\title{
Alleviation of spinal cord injury by Ginkgolide $B$ via the inhibition of STAT1 expression
}

\author{
J.L. Zheng, B.S. Li, X.C. Cao, W.K. Zhuo and G. Zhang \\ Department of Orthopedics Surgery, Jinan Military General Hospital, Jinan, \\ Shandong, China \\ Corresponding author: J.L. Zheng \\ E-mail: zhjinlongl@126.com
}

Genet. Mol. Res. 15 (2): gmr. 15027673

Received September 18, 2015

Accepted December 8, 2015

Published June 17, 2016

DOI http://dx.doi.org/10.4238/gmr.15027673

\begin{abstract}
Ginkgolide B has been known to inhibit cell apoptosis by modulating multiple cytokines and plays an important role in neuroprotection. Signal transducer and activator of transcription 1 (STAT1) has been studied in a spinal cord injury (SCI) model. However, the role of Ginkgolide B in SCI treatment remains unclear. This study investigated the potential mechanism of Ginkgolide B using an SCI rat model. SD rats were used to generate an SCI model followed by Ginkgolide B injection ( $4 \mathrm{mg} / \mathrm{kg}$ ) for 14 days. Spinal cord tissue samples were examined using hematoxylin and eosin (H\&E) staining. The expression of STAT1 was determined by western blot. Using a dyskinesia scale, intervention with Ginkgolide B significantly decreased the severity of SCI. H\&E staining revealed less nuclear condensation and cell necrosis in SCI rats after treatment with Ginkgolide B. STAT1 expression was significantly increased in SCI model rats, but was lower after Ginkgolide B treatment. Therefore, Ginkgolide B can effectively inhibit STAT1 expression and alleviate SCI.
\end{abstract}

Key words: Ginkgolide B; Signal transducer and activator of transcription 1; Spinal cord injury 


\section{INTRODUCTION}

Spinal cord injury (SCI) can be caused by multiple factors including trauma and degenerative disease. Although significant progress has been made regarding its diagnosis, treatment and rehabilitation, a cure for SCI is still a major hurdle in neurosurgery (Dasari et al., 2008; Zhao et al., 2012). Primary and secondary injuries occur sequentially in SCI. Primary injury occurs within a short-time window and causes irreversible physical damage to neural tissues. On the other hand, secondary injury leads to a series of pathological changes such as focal ischemia, tissue edema, and inflammation (Park et al., 2008).

During the SCI process, signal transduction plays an important role as a complexregulatory network is involved including mitogen-activated protein kinase (MAPK), phosphoinositide-3-kinase (PI3K)/Akt, glycogen synthase kinase $3 \beta$ (GSK-3 $\beta$ ), and Janus kinase (JAK)/signal transducer and activator of transcription 1 (STAT1). Among these wellstudied pathways, STAT1 plays a critical role in signal transduction as a transcription factor by binding specifically onto surface receptors, resulting in its phosphorylation and nuclear translocation (Calapai et al., 2000; Wang et al., 2006; Dominguez et al., 2010).

Extracts of ginkgo biloba have significant pharmaceutical value and have been used in both basic and clinical studies, particularly with respect to the nervous system. Animal models have shown the alleviation of ischemia-induced neural injuries by ginkgo biloba extracts by their inhibition of nitric oxide synthase. Clinical studies also revealed the remarkable role of extracts in treating Alzheimer's disease and other brain disorders (Tchantchou et al., 2007). A recent in vivo study showed the protection of spinal cord neurons by the inhibition of cytosolic phospholipase A2 (cPLA2) and caspase-3 expression, thus depressing cell apoptosis (Zhao et al., 2011). As the major component of ginkgo biloba extracts, Ginkgolide B is a potent platelet activating factor and can penetrate the blood-brain barrier (Zhao et al., 2011). The purpose of this study was to investigate the mechanism of Ginkgolide B in alleviating SCI.

\section{MATERIAL AND METHODS}

\section{SCI animal model}

Rats were provided by the Shandong University Animal Experiment Center (license key: SYXK-2013-1239). A total of $30 \mathrm{SD}$ rats were anesthetized and an area of $9 \mathrm{~mm}^{2}$ was exposed around the T10 spine process as previously described (Vawda and Fehlings, 2013). The spinal cord was clipped for $3 \mathrm{~s}$ to generate SCI. A spastic tail movement and retraction of hind limbs followed by paralysis indicated the successful SCI model. The sham group received anesthesia, but did not underwent any surgery. Rats were used for all experiments and all procedures were approved by the Animal Ethics Committee of Jinan Military General Hospital.

The motor function of rats was evaluated 1, 3, 7, and 14 days after surgery. Using the Basso, Beattie and Bresnahan (BBB) scale, each animal was observed walking for 15-30 min. A score higher than 20 was considered normal walking; a score less than 8 was identified as motor dysfunction; and a score of 0 represented complete loss of motor function. Each animal also underwent IPT, the ramp test which is another method of motion detection. 


\section{Hematoxylin and eosin (H\&E) staining}

Spinal cord segment tissues were fixed in formalin overnight, followed by serial dehydration in $70 \%$ ethanol ( $3 \mathrm{~h}$ ), $80 \%$ ethanol $(3 \mathrm{~h}), 95 \%$ ethanol $(2 \mathrm{~h}), 100 \%$ ethanol $(1.5 \mathrm{~h}$, twice) and xylene $\left(0.5 \mathrm{~h}\right.$, twice). After immersion in paraffin $\left(1 \mathrm{~h}\right.$ at $55^{\circ} \mathrm{C}$ and $2 \mathrm{~h}$ at $\left.60^{\circ} \mathrm{C}\right)$, tissues were embedded and sectioned in to $3-\mu \mathrm{m}$ slices. Using xylene and gradient ethanol for dewaxing, slices were sequentially stained in hematoxylin $(1 \mathrm{~min})$ and eosin $(10 \mathrm{~s})$, and were mounted on glass slides. The pathological changes were evaluated by three independent observers in a blinded manner.

\section{Western blotting}

Total proteins were extracted from spinal cord tissues using protein extraction kit (Beyotime Biotechnology, Shanghai, China) and equal amounts of protein from each sample were loaded onto SDS-PAGE for separation. The electrophoresis was carried out at a voltage of $80 \mathrm{~V}$ for $1 \mathrm{~h}$ and $120 \mathrm{~V}$ until the die-front reached the bottom of the gel. Proteins were then transferred to PVDF membrane under a $250-\mathrm{mA}$ electrical field for $90 \mathrm{~min}$. After gentle rinsing in TBS-T, the membrane was blocked in 5\% skim milk powder for $1 \mathrm{~h}$. Rabbit anti-rat STAT1 primary antibody in 1:100 dilution (Santa Cruz, CA, USA) was applied for overnight incubation. The next day, the membrane was rinsed in TBS-T three times, followed by incubation with goat anti-rabbit secondary antibody (1:100 dilution, Santa Cruz). ECL chromogenic substrate was then used to develop the membrane.

\section{Statistical analysis}

The SPSS 11.0 package software (Chicago, IL, USA) was used to process all collected data. The Student $t$-test was used to compare means between groups. Pearson analysis revealed correlation between parameters. A statistical significance was defined when $\mathrm{P}<0.05$.

\section{RESULTS}

\section{Ginkgolide B facilitates motor function recovery after SCI}

Significant motor dysfunction can be observed by the naked eye after SCI model generation. The sham group had no significant change in motor function. IPT score revealed elevated motor function in SCI rats with Ginkgolide B intervention compared to the SCIuntreated group $(\mathrm{P}<0.05$; Figure 1A). BBB score showed consistent results as Ginkgolide $\mathrm{B}$ improved motor score significantly with longer treatment times $(\mathrm{P}<0.05$; Figure $1 \mathrm{~B})$.

\section{Cell morphology changes in SCI}

In sham rats, we found clear cell shape with normal nuclear morphology. In SCI model animals, nuclear condensation was observed at day 3 with the occurrence of necrotic cells at day 14. At the same time points, we found less nuclear condensation and cell necrosis in rats with Ginkgolide B treatment (Figure 2). These results support the hypothesis that cellular integrity is maintained with Ginkgolide B treatment after induction of SCI. 

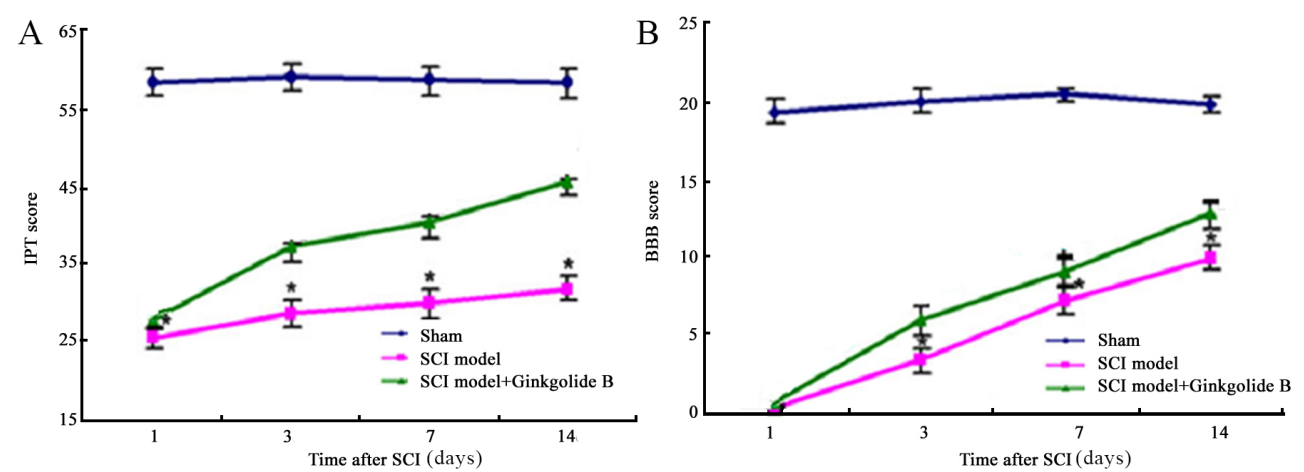

Figure 1. Motor function of rats after SCI. A. IPT score was lower in model rats but was higher after Ginkgolide B application. B. BBB score showed similar patterns. In both scenarios, Ginkgolide B resulted in significant improvements with longer treatment. $* \mathrm{P}<0.05$ compared to sham group.

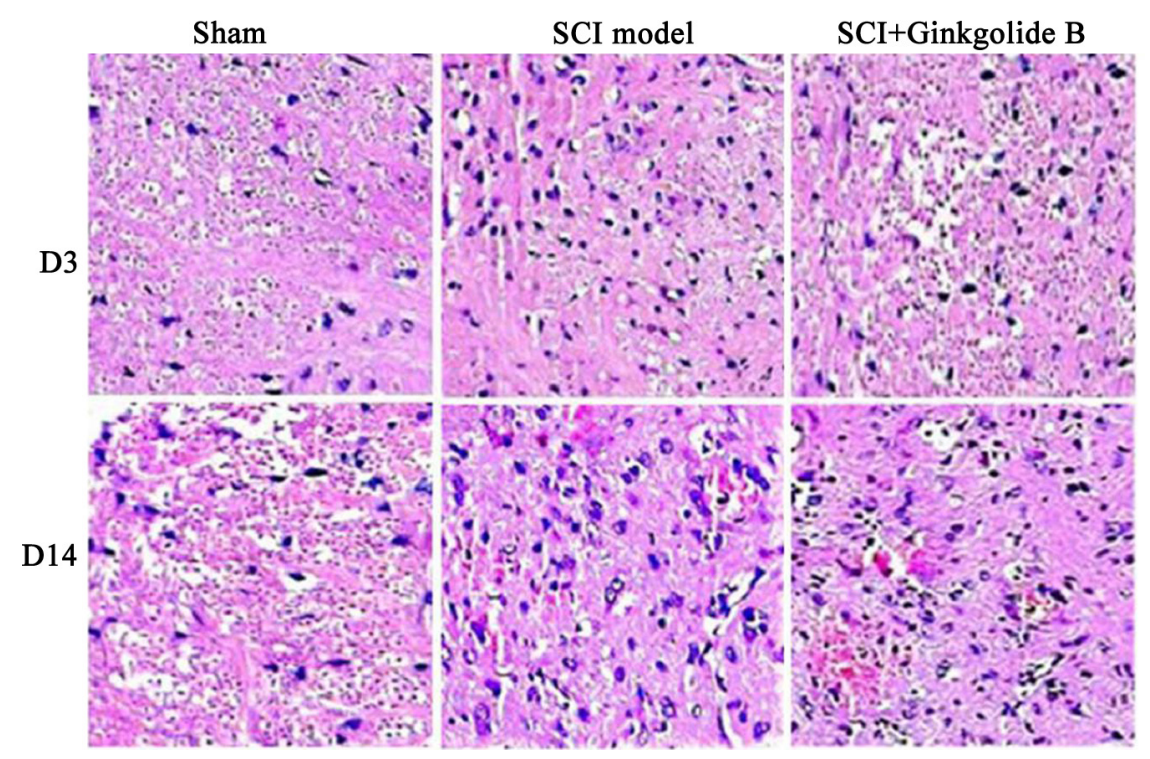

Figure 2. Spinal cord tissue morphology as shown by H\&E staining. Sham animals (left panels) had clear nuclei without necrotic cells. SCI model (middle panels) had significantly increased nuclear condensation and cell necrosis. The application of Ginkgolide B (right panel) alleviated tissue injury compared to SCI model group. Cells are shown on day 3 (D3) and day 14 (D14).

\section{Ginkgolide B inhibits STAT1 expression}

As STAT1 is a major transcription factor expressed during signal transduction, we tested STAT1 expression in all animals. Specifically, the nuclear fraction of proteins was quantified, as phosphorylated STAT1 migrates into the nucleus to initiate transcription. Western blotting results showed that STAT1 activity was potentiated in SCI model group. Treatment with Ginkgolide B reduced STAT1 activity to levels similar to the sham group $(\mathrm{P}<$ 0.05). These results are shown in Figure 3. 


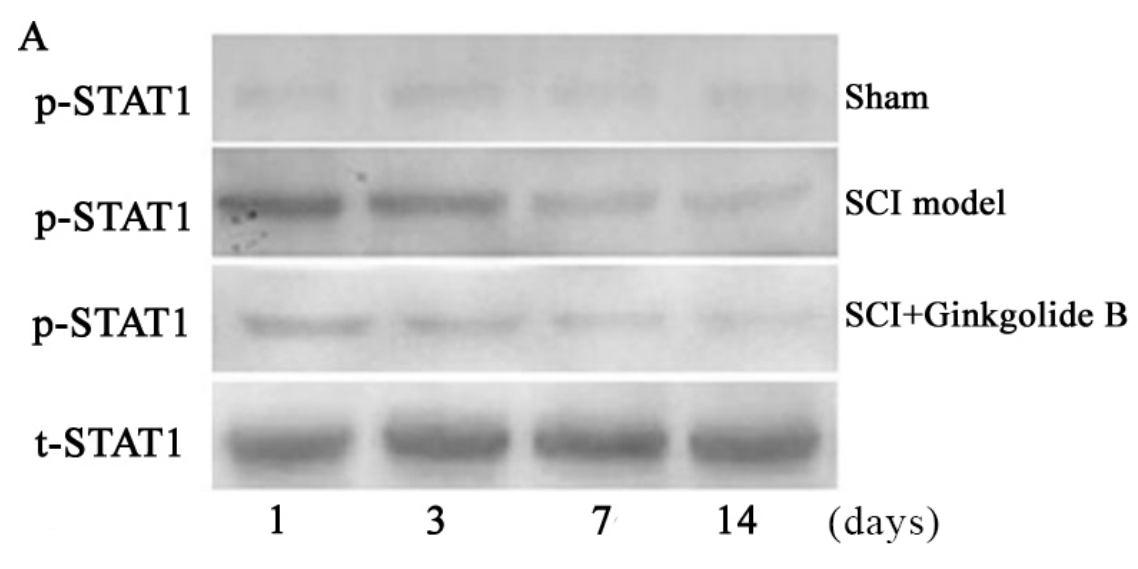

B

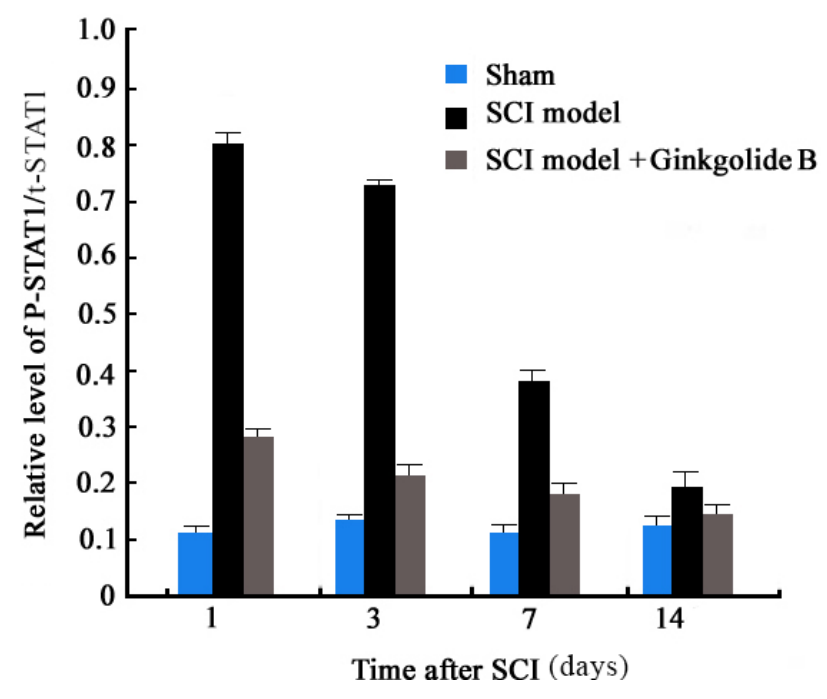

Figure 3. Expression of phosphorylated STAT1 (p-STAT1) and total STAT1 (t-STAT1) in rat spinal cords. A. Western blot and B. quantitation of p-STAT1/t-STAT1 at different time points.

\section{DISCUSSION}

SCI is mainly caused by traffic accidents, sports injuries and other traumas, and can cause severe neuropathological damage and neural dysfunction (Massieu et al., 2004). Secondary injury during SCI can cause apoptosis of neurons and glial cells, increasing the permeability of the spinal cord barrier and increasing neural reaction response time (Lin et al., 2008; Yoshimura et al., 2011). The secondary inflammation reaction in the spinal cord is even more severe than in brain tissue. Some studies have tried to manipulate the composition of inflammatory cells in mouse models of SCI but did not obtain satisfactory results (Shi et al., 2010; Lukáš et al., 2011), suggesting the necessity for the development of alternative treatments for secondary SCI. 
The JAK/STAT signaling pathway is activated immediately after SCI and is accompanied with morphological changes and cell apoptosis (Cayli et al., 2004). Other studies described the activation of STAT1 in cerebral focal ischemia and brain damage and many potent antioxidants exert their protective functions via inhibition of STAT1 phosphorylation and nuclear translocation (Choi et al., 2011; Xu et al., 2011; Rong et al., 2012). The protective role of Ginkgolide B in SCI has not been reported; therefore, we hypothesized that Ginkgolide B might exert its protection of the spinal cord through the STAT1 pathway. This is based on evidence showing the alteration of important transcription factors, such as STAT1 and tumor growth factor $\beta$ (TGF- $\beta$ ), in other injured tissues such as bone (Han et al., 2012; Ishii et al., 2013). Thus, we hypothesized that Ginkgolide B might exert similar effects through STAT1 in SCI.

Our study showed the important role of STAT1 in spinal cord tissues, where STAT1 is dephosphorylated under normal physiological conditions. The phosphorylation of STAT1 was initiated immediately after SCI induction and reached a peak 1 day after injury, following which it gradually decreased. These results suggested the activation of STAT1 after SCI, leading to nuclear condensation and cell necrosis and resulting in a lower motor score. Intervention of SCI with Ginkgolide B significantly decreased STAT1 phosphorylation levels, decreased nuclear condensation and cell necrosis, and initiated partial recovery of motor function.

In summary, this study demonstrated the facilitation of spinal cord function occurs through Ginkgolide B, possibly via STAT1 activation. Our results provide more insights regarding the clinical treatment of SCI.

\section{Conflicts of interest}

The authors declare no conflict of interest.

\section{ACKNOWLEDGMENTS}

We thank the anonymous reviewers for reviewing this manuscript.

\section{REFERENCES}

Calapai G, Crupi A, Firenzuoli F, Marciano MC, et al. (2000). Neuroprotective effects of Ginkgo biloba extract in brain ischemia are mediated by inhibition of nitric oxide synthesis. Life Sci. 67: 2673-2683. http://dx.doi.org/10.1016/ $\underline{\mathrm{S} 0024-3205(00) 00858-4}$

Cayli SR, Kocak A, Yilmaz U, Tekiner A, et al. (2004). Effect of combined treatment with melatonin and methylprednisolone on neurological recovery after experimental spinal cord injury. Eur. Spine J. 13: 724-732. http://dx.doi.org/10.1007/ $\underline{\mathrm{s} 00586-003-0550-\mathrm{y}}$

Choi JK, Kim KH, Park H, Park SR, et al. (2011). Granulocyte macrophage-colony stimulating factor shows anti-apoptotic activity in neural progenitor cells via JAK/STAT5-Bcl-2 pathway. Apoptosis 16: 127-134. http://dx.doi.org/10.1007/ $\underline{\text { s10495-010-0552-2 }}$

Dasari VR, Spomar DG, Li L, Gujrati M, et al. (2008). Umbilical cord blood stem cell mediated downregulation of fas improves functional recovery of rats after spinal cord injury. Neurochem. Res. 33: 134-149. http://dx.doi.org/10.1007/ $\underline{\text { s11064-007-9426-6 }}$

Dominguez E, Mauborgne A, Mallet J, Desclaux M, et al. (2010). SOCS3-mediated blockade of JAK/STAT3 signaling pathway reveals its major contribution to spinal cord neuroinflammation and mechanical allodynia after peripheral nerve injury. J. Neurosci. 30: 5754-5766. http://dx.doi.org/10.1523/JNEUROSCI.5007-09.2010

Han X, Lu M, Wang S, Lv D, et al. (2012). Targeting IKK/NF-kB pathway reduces infiltration of inflammatory cells and apoptosis after spinal cord injury in rats. Neurosci. Lett. 511: 28-32. http://dx.doi.org/10.1016/j.neulet.2012.01.030 
Ishii H, Tanabe S, Ueno M, Kubo T, et al. (2013). ifn- $\gamma$-dependent secretion of IL-10 from Th1 cells and microglia/ macrophages contributes to functional recovery after spinal cord injury. Cell Death Dis. 4: e710. http://dx.doi. org $/ 10.1038 /$ cddis.2013.234

Lin Y, Wang R, Wang X, He RR, et al. (2008). Effects of ginkgolide B on neuronal discharges in paraventricular nucleus of rat hypothalamic slices. Neurosci. Bull. 24: 345-350. http://dx.doi.org/10.1007/s12264-008-2716-4

Lukáš R, Zýková I, Barsa P and Srám J (2011). [Current role of methylprednisolone in the treatment of acute spinal cord injury]. Acta Chir. Orthop. Traumatol. Cech. 78: 305-313.

Massieu L, Morán J and Christen Y (2004). Effect of Ginkgo biloba (EGb 761) on staurosporine-induced neuronal death and caspase activity in cortical cultured neurons. Brain Res. 1002: 76-85. http://dx.doi.org/10.1016/j. brainres.2003.12.018

Park KK, Liu K, Hu Y, Smith PD, et al. (2008). Promoting axon regeneration in the adult CNS by modulation of the PTEN/ mTOR pathway. Science 322: 963-966. http://dx.doi.org/10.1126/science.1161566

Rong W, Wang J, Liu X, Jiang L, et al. (2012). Naringin treatment improves functional recovery by increasing BDNF and VEGF expression, inhibiting neuronal apoptosis after spinal cord injury. Neurochem. Res. 37: 1615-1623. http:// dx.doi.org/10.1007/s11064-012-0756-7

Shi C, Wu F, Yew DT, Xu J, et al. (2010). Bilobalide prevents apoptosis through activation of the PI3K/Akt pathway in SH-SY5Y cells. Apoptosis 15: 715-727. http://dx.doi.org/10.1007/s10495-010-0492-x

Tchantchou F, Xu Y, Wu Y, Christen Y, et al. (2007). EGb 761 enhances adult hippocampal neurogenesis and phosphorylation of CREB in transgenic mouse model of Alzheimer's disease. FASEB J. 21:2400-2408. http://dx.doi. org/10.1096/fj.06-7649com

Vawda R and Fehlings MG (2013). Mesenchymal cells in the treatment of spinal cord injury: current \& future perspectives. Curr. Stem Cell Res. Ther. 8: 25-38. http://dx.doi.org/10.2174/1574888XX11308010005

Wang GX, Cao FL and Chen J (2006). Progress in researches on the pharmaceutical mechanism and clinical application of Ginkgo Biloba extract on various kinds of diseases. Chin. J. Integr. Med. 12: 234-239. http://dx.doi.org/10.1007/ BF02836532

Xu J, Qin X, Wang J, Zhang S, et al.; Society of Surgery; Chinese Medical Association; Committee of Colorectal Cancer, Chinese Anti-cancer Association (2011). Chinese guidelines for the diagnosis and comprehensive treatment of hepatic metastasis of colorectal cancer. J. Cancer Res. Clin. Oncol. 137: 1379-1396. http://dx.doi.org/10.1007/ $\underline{\text { s00432-011-0999-8 }}$

Yoshimura K, Ueno M, Lee S, Nakamura Y, et al. (2011). c-Jun N-terminal kinase induces axonal degeneration and limits motor recovery after spinal cord injury in mice. Neurosci. Res. 71: 266-277. http://dx.doi.org/10.1016/j. neures.2011.07.1830

Zhao T, Qi Y, Li Y and Xu K (2012). PI3 Kinase regulation of neural regeneration and muscle hypertrophy after spinal cord injury. Mol. Biol. Rep. 39: 3541-3547. http://dx.doi.org/10.1007/s11033-011-1127-1

Zhao Z, Liu N, Huang J, Lu PH, et al. (2011). Inhibition of cPLA2 activation by Ginkgo biloba extract protects spinal cord neurons from glutamate excitotoxicity and oxidative stress-induced cell death. J. Neurochem. 116: 1057-1065. http:// dx.doi.org/10.1111/j.1471-4159.2010.07160.x 